\title{
Cohort Selection of Specific User Using Max-Min-Centroid- Cluster (MMCC) Method to Enhance the Performance of a Biometric System
}

\author{
Jogendra Garain ${ }^{1}$, Ravi Kant Kumar ${ }^{2}$, Goutam Sanyal ${ }^{3}$ and Dakshina Ranjan \\ $\mathrm{Kisku}^{4}$ \\ 1,2,3,4 Department of Computer Science and Engineering \\ National Institute of Technology Durgapur \\ Durgapur - 713209, West Bengal, India \\ \{jogs.cse, vit.ravikant,nitgsanyal,drkisku\}@gmail.com
}

\begin{abstract}
Selection of cohort models plays a vital role to increase the accuracy of a biometric authentication system as well as to reduce the computational cost. This paper proposes a novel approach for cohort selection called Max-Min-CentroidCluster (MMCC) method. The clusters of cohorts are generated by K-means clustering technique. The union of the clusters having largest and smallest centroid value is taken as cohort subset. The cohort scores, after normalization using different cohort based score normalization techniques, are used in authentication process of the system. Evaluation has been carried out on FEI face datasets. The performance of this novel methodology is analyzed using T-norm and Aggarwal (max rule) normalization techniques. Experimental results exhibit the efficacy of the proposed method.
\end{abstract}

Keywords: Cohort Model, Authentication System, Cohort Selection, Max-MinCentroid-Cluster, K-means, Normalization Technique

\section{Introduction}

Biometric authentication system often requires identity verification of individuals to avoid susceptible attacks and gain access to the systems protecting invaluable and important files. It is proved to be a very challenging topic in identity management and machine vision because of its frequent applications in high security zones which requires cent percent accuracy. That is why, present day scenario demands computationally efficient approaches in addition to traditional biometric systems to which the researchers are heavily being attracted. As a result, novel techniques are being evolved toward the cohort selection and score normalization techniques [1][9] which can fulfill the various requirements of biometric systems used in many organizations by enhancing their performance.

From the perspective of cohort selection method, a biometric system is having all the templates other than claimed identity are called cohort template. A cohort score is the matching score between a cohort template and the query template. The similarity score between the query template and claimed template is normalized with the help of obtained cohort scores. If there are $N$ no of templates in a system's database then there will be $N-1$ cohort templates. So if the query template is compared with all the cohort templates it will be computationally very expensive for a large database. Looking for the solution of this problem without degrading the system's performance brings the researcher's attentions toward the proper selection of cohort subset. Researchers are also working on multimodal biometric systems 
[10-12] which outperform mono-modal biometric systems. However, with the increase of number of modalities and number of subjects in database, computational cost is proportionally increased. Many researchers have indicated that cohort selection can be a way out of this particular problem. Aggarwal et.al., [7, 13], Amin Merati et. al., [1] have shown that after applying appropriate cohort selection method a biometric system can be outperformed other systems which have not used cohort selection strategy. Tistarelli et. al., [2] have been worked on unconstrained face recognition on the use of discriminative cohort score. Amin Merati et. al.,[1] have also specified the computational cost to decrease by half of the total cost. Our target in this paper to select a suitable set of imposter (cohort) to normalize the similarity scores using the cohort subset to improve the system performance by minimizing the effects of extrinsic and intrinsic factors which always degrade the authentication system's performance by varying the raw similarity scores.

This paper proposes a novel methodology for cohort subset selection called MaxMin-Centroid-Cluster (MMCC) where the set of cohort scores are clustered using Kmeans clustering algorithm [14] and cluster centre initialization algorithm [15] and then union of non-match templates whose scores are contained by the clusters having maximum and minimum centroid value are taken as cohort subset. In other words it can be said that the cohorts which are either very close or very far in respect of similarity score are taken into the account of cohort subset. Amin Merati et. al., [16] have stated that the most similar and most dissimilar cohorts have discriminative characteristics.

The main contribution of this work is as follows

- In some previous work discussed in [13] stated that the cohorts have been chosen from the enrolled identity. However, in the proposed work, cohorts have been taken from a different dataset called training dataset.

- The training dataset contains the smiling faces of the same subjects enrolled into database. This is one more significant contribution of this paper which considers the effect of the expression change on the normalized score.

- Unlike some prior work [13] where a fixed number of cohorts have been taken into account, the subset has been given the flexibility to vary from 10 to 40 in the proposed work.

The paper is arranged as following. Section 2 describes the proposed cohort selection method. Section 3 formulate and calculate cohort-based normalized score using two most popular normalization techniques. Section 4 shows the results of experiments explaining the comparison and usefulness of the proposed method. Finally, Section 5 concludes and states the future scope.

\section{Cohort Selection}

The most crucial and important issue of the proposed strategy is to select a suitable subset of non-matched template (cohort) from the training dataset of each subject from enrolled dataset. The novel algorithm for cohort subset selection is presented below.

\footnotetext{
Algorithm 1: Cohort Subset Selection

1. Generate $\mathrm{n}$ number of clusters $\left\{C_{1}, C_{2}, \ldots, C_{n-1}, C_{n}\right\}$ with $\delta$ no of cohort scores in each cluster using k-means clustering algorithm.

2. Find out $\psi_{j}, j=1,2, \ldots, \mathrm{n}$ where $\psi_{\mathrm{j}}$ is the centroid of $j^{\text {th }}$ cluster.

3. Find $\psi_{\max }=\max \left\{\psi_{1}, \psi_{2}, \ldots, \psi_{\mathrm{n}}\right\}$ and $\psi_{\min }=\min \left\{\psi_{1}, \psi_{2}, \ldots, \psi_{\mathrm{n}}\right\}$

4. Select $\left\{C_{\mathrm{x}} U C_{\mathrm{y}}\right\}$ as required subset of cohort where $\psi_{\mathrm{x}}=\psi_{\max }$ and $\psi_{\mathrm{y}}=\psi_{\min }$
} 
Figure 1 shows the no of cohort selected for each subjects through the proposed methodology. Eight of the selected cohort for four different subjects has been depicted in Figure 2. In each row the first face (normal face) is the enrollee and the other 8 faces (smiling faces) in column 2 to 9 are some of the selected cohorts of that enrolled face.

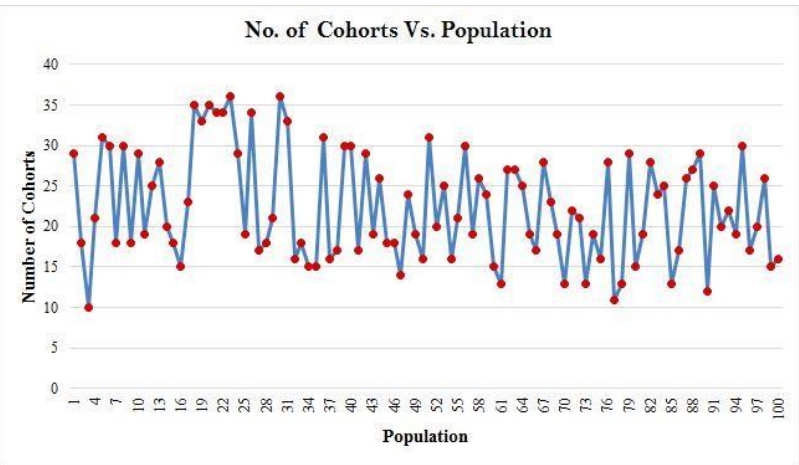

Figure 1. Number of Cohorts per Enrolled Subjects

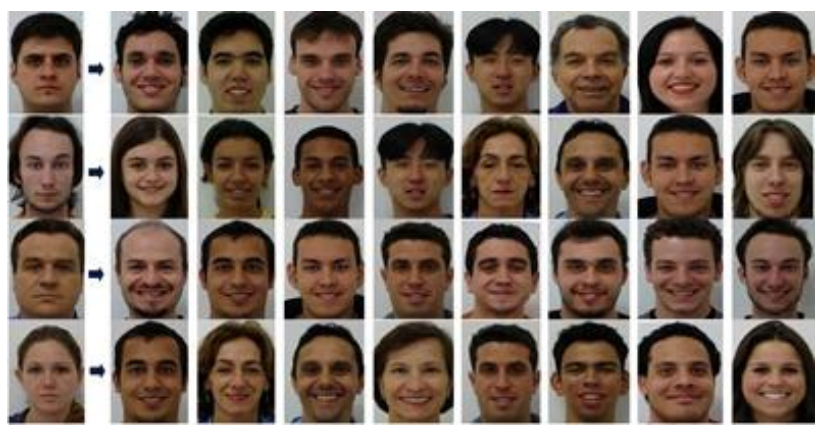

Figure 2. Column 1 Enrolled Sample, Column 2-9: Selected Cohorts from Training Sample

\section{Score Normalization using Different Normalization Techniques}

In the previous section we have discussed cohort selection in Section 2. In this section, we use two well-known cohort score normalization techniques and their mathematical formulae to be needed for obtaining scaled-up cohort scores.

\subsection{T-Norm Normalization:}

The most popular and commonly used cohort normalization technique is T-norm which is based on two parameters mean $(\mu)$ and variance $(v)$ determined from the matching scores. The mean $(\mu)$ indicates the mean value of all the cohort scores. In this technique the statistic is estimated online and it is test dependent method but it reduces the overlap between imposter and true score distribution of each client. The drawback of this method is additional test stage computation in scoring the cohort models. The normalized score $\left(s_{n}\right)$ is calculated using the mathematical formulation given below. 


$$
\begin{gathered}
(S n)=\frac{g-\mu}{\sigma}, \text { where } g=\text { genuine score, mean }(\mu)=\frac{1}{n} \sum_{i=1}^{n} \lambda i \\
\text { variance }(v)=\frac{1}{n} \sum_{i=1}^{n}(\lambda i-\mu)^{2} \text { and } \text { standard deviation }(\sigma)=\sqrt{v}, \lambda=\text { cohort score }
\end{gathered}
$$

\subsection{Aggarwal (max rule) Normalization:}

Aggarwal [7, 13] et. al., explained the biometric authentication as a statistical test where the claimed identity is considered as always true. In this method number of computation is lesser than T-norm because the greatest cohort score is found out first then the ratio of this obtained highest score and the genuine matching score is taken as test statistics, other scores are not considered. The equation is shown below.

$$
\text { Normalized score }\left(S_{A g}\right)=\frac{g}{\max \left\{\lambda_{i}\right\}_{i=1 \text { to } n}}
$$

After the proper selection of the cohort subset the matching scores are normalized using T-normalization using Equation (1) and Aggarwal (max rule) normalization using Equation (2). Figure 3 shows the how the raw matching scores and normalized scores (a) between enrolled and training dataset (b) enrolled and testing dataset are deviated. Figure 3(c) shows the raw similarity scores (without any normalization) between enrolled and testing dataset. Later in Section 4, the effects of our proposed technique have been shown.

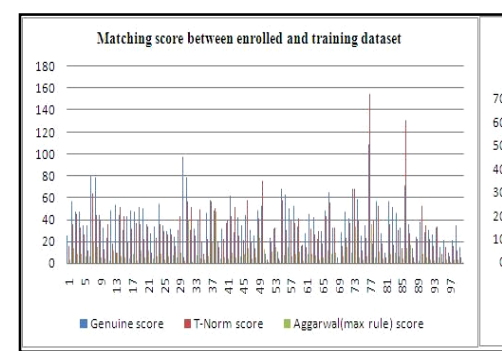

(a)

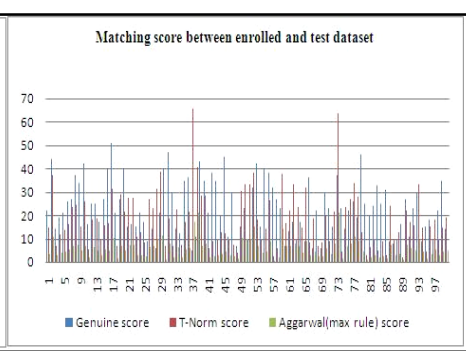

(b)

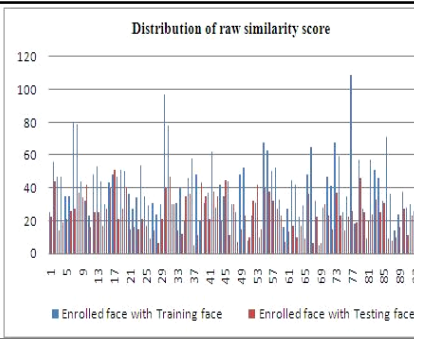

(c)

Figure 3. (a) Score Distribution between Enrolled and Training Dataset, (b) Score Distribution between Enrolled and Testing Dataset, (c) Distribution of Raw Similarity Score between Claimed and Query Face

\section{Experimental Evaluation}

\subsection{Database and Experimental Setup}

The experiment is concerned with the problem of personal identity verification. Face biometric information is used to check the claimed identity of an individual. Performance evaluation has been carried out using the FEI database which is made up of 14 samples of each of the 200 subjects. In order to perform the experiment, 100 subjects has been taken with three samples (three shots), one normal face, one smiling face and other one is normal face taken in low lighting condition, which are used as enrolled dataset, training dataset and test dataset respectively shown in Figure 4 . The resolution of all images is fixed at 260x360 pixels. In the proposed work, we use standard SIFT point matching [17] algorithm to generate matching scores. 


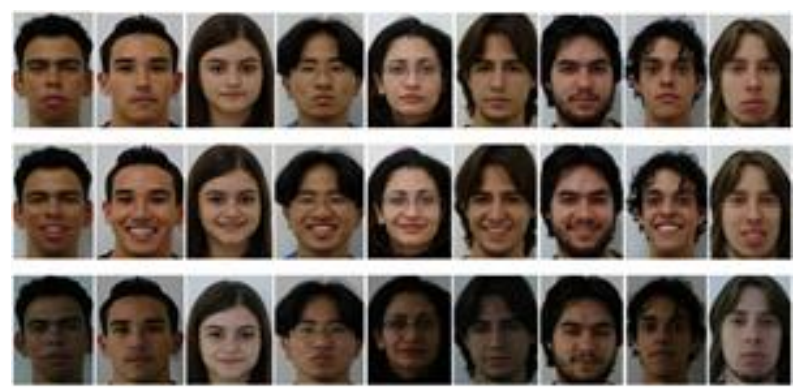

Figure 4. Enrolled Dataset (First Row), Training Dataset (Second Row), Test Dataset (Third Row)

\subsection{Experimental Results and Performance Evolution}

Here the cropped faces are taken as input to the SIFT matching algorithm[17] to find out the raw matching score between the enrolled faces (normal face) and the training faces (smiling face).Then scores are normalized using $\mathrm{T}$-norm and Aggarwal(max rule) normalization and the normalized scores are associated with the template profile. The performance of the verification system using the proposed cohort selection method has been depicted in Figure 5 where ROC curve shows the trade-off between FAR (false accept rate) and FRR (false reject rate) for T-norm and Aggarwal(max rule) normalizations and the performance of the proposed system has been drawn out in terms of accuracy in Table 1. It gives a clear picture where Tnorm outperforms Aggarwal(max rule) normalization. This happens because the later method deals with only one enrolled template, the maximum among the cohorts whereas the former one considers all the cohorts to find out the mean and variance. Since the query template is not compared here with all the cohorts of the claimed template, only the cohorts of the subset selected by the proposed algorithm are compared, the maximum no of comparison required is 40 . So the computational cost is reduced as much as $2 / 5^{\text {th }}$ of total cost.

Table 1. FAR (False Accept Rate), FRR (False Reject Rate), EER (equal error rate) and GAR (Genuine Accept Rate) in Addition to Overall Accuracy of the Proposed System are Shown

\begin{tabular}{|c|c|c|c|c|c|}
\hline $\begin{array}{c}\text { Normalization } \\
\text { Method }\end{array}$ & FAR (\%) & FRR (\%) & EER (\%) & GAR (\%) & ACCURACY (\%) \\
\hline T - Norm & 7.87 & 3.47 & 5.67 & 96.53 & $\mathbf{9 5 . 3 3}$ \\
\hline Aggarwal(Max Rule) & 12.25 & 7.53 & 9.89 & 92.47 & $\mathbf{9 0 . 1 1}$ \\
\hline
\end{tabular}




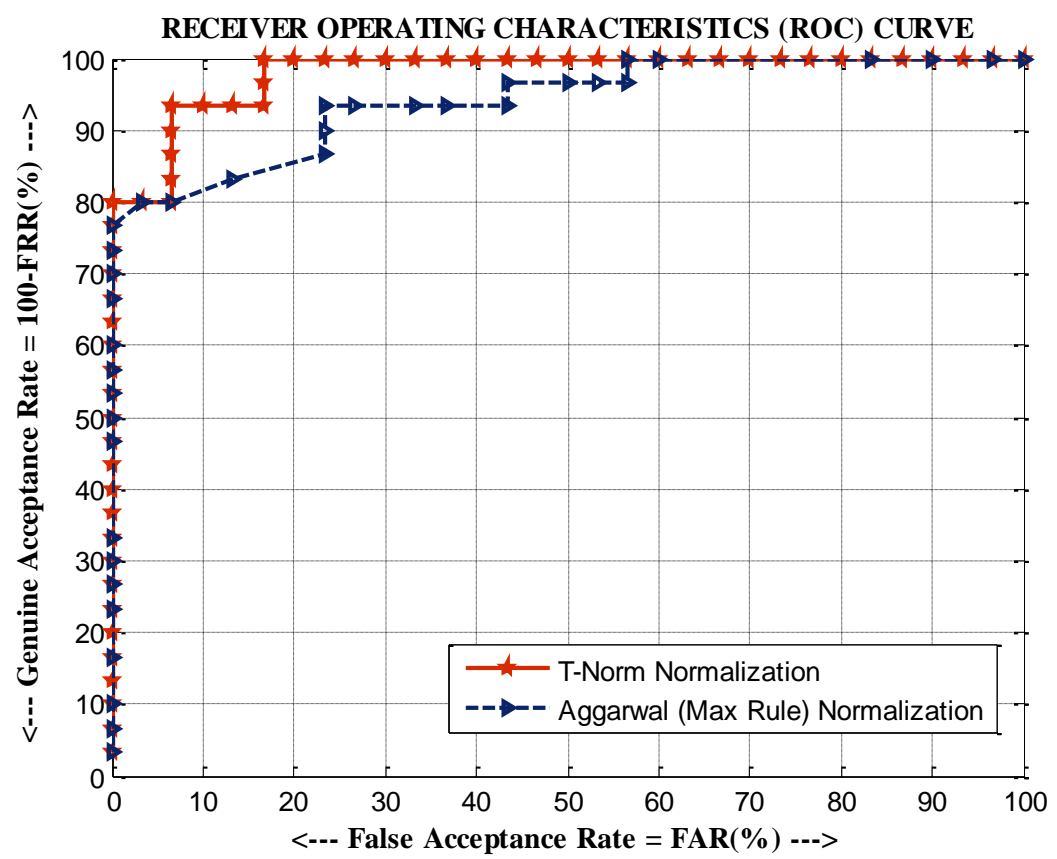

Figure 5. Receiver Operating Characteristics (ROC) Curve showing FAR (\%) and FRR (\%) for T-Norm and Aggarwal (max rule) Normalization

\section{Conclusion and Future Work}

This paper reports a biometric system which makes use of user specific cohort selection method known as Max-Min-Centroid-Cluster (MMCC) technique. Experiment has been carried out only on frontal cropped faces because the SIFT matching algorithm used here gives well performance on the frontal faces. But this method for cohort selection can be used for experiment with faces of different pose and expressions with other matching algorithms. This cohort selection approach can also be extended for multimodal biometric. In our experimental work only one template per subject is enrolled in the database so there is another scope to enrich the performance by enrolling multiple templates of each subject. Though we focus only on face biometric, in case of other biometrics, the proposed methodology can also be applied without much modification.

\section{References}

[1] Merati, N. Poh and J. Kittler, User-Specific Cohort Selection and Score Normalization for Biometric Systems, IEEE Transactions on Information Forensics and Security, 7(4) (2012).

[2] M. Tistarelli, Y. Sun and N. Poh, On the Use of Discriminative Cohort Score Normalization for Unconstrained Face Recognition, IEEE Transactions on Information Forensics and Security, 9(12), 2063-2-75 (2014)

[3] J. M. Colombi, D. W Ruck, T. R. Anderson, S. K. Rogers and M. Oxley, Cohort Selection and Word Grammar Effects for Speaker Recognition,Proceedings of the IEEE International Conference on Acoustics, Speech, and Signal Processing (ICASSP'96),(1996), 85-88.

[4] N. Poh, A. Ross, W. Li and J. Kittler, User-Specific and Selective Multimodal Biometric Fusion Strategy by Ranking Subjects, Pattern Recognition, 46(12), 3341-57 (2013).

[5] A. Kumar, Incorporating Cohort Information for Reliable Palmprint Authentication, Proceedings of the $6^{\text {th }}$ Indian Conference on Computer Vision, Graphics and Image Processing, (2008), 583-590.

[6] P. Wang, Q. Ji and J. L. Wayman, Modeling and Predicting Face Recognition System Performance Based on Analysis of Similarity Scores,IEEE Transactions on Pattern Analysis and Machine Intelligence, 29(4)(2007).

[7] G. Aggarwal, N. Ratha and R. M. Bolle, Biometric Verification: Looking beyond Raw Similarity Scores,Proceedings of the IEEE Computer Society Workshop on Biometrics (CVPR),(2006). 
[8] R. Auckenthaler, M. Carey and H. Lloyd-Thomas, Score Normalization for Text-independent Speaker Verification Systems, Digital Signal Processing, 10, 42-54, (2000).

[9] S. Tulyakov, Z. Zhang and V. Govindaraju, Comparison of Combination Methods Utilizing Tnormalization and Second Best Score Model, Proceedings of the IEEE Conference on Computer Vision and Pattern Recognition Workshop, (2008),Los Alamitos, CA, 1-5.

[10] A. Jain, K. Nandakumarand A. Ross, Score Normalization in Multimodal Biometric Systems, Pattern recognition, 38(12), 2270-2285,(2005).

[11] M. He, S. J. Horng, P. Fan, R. S. Run, R. J. Chen, J. L. Lai \& K. O. Sentosa, Performance evaluation of score level fusion in multimodal biometric systems. Pattern Recognition, 43(5), 1789-1800, (2010).

[12] J. Bhatnagar, \& A. Kumar, On estimating performance indices for biometric identification. Pattern Recognition, 42(9), 1803-1815(2009).

[13] G. Aggarwal, N. K. Ratha, R. M. Bolle, and R. Chellappa, Multibiometric cohort analysis for biometric fusion, in IEEE International Conference, Acoustics, Speech and Signal Processing, pp. 5224-5227, (2008).

[14] T. Kanungo, D. M. Mount N. S. Netanyahu, C. D. Piatko, R. Silverman \& A.Y. Wu, An efficient kmeans clustering algorithm: Analysis and implementation. Pattern Analysis and Machine Intelligence, IEEE Transactions on, 24(7), 881-892 (2002).

[15] S. S. Khan, \& A. Ahmad, Cluster center initialization algorithm for K-means clustering, Pattern recognition letters, 25(11), 1293-1302(2004).

[16] A. Merati, N. Poh, and J. Kittler, Extracting discriminative information from cohort models, in Proc. 4th IEEE Int. Conf. Biometrics: Theory Applications and Systems (BTAS), Washington, DC, pp. 1-6, Sep. (2010).

[17] D. G. Lowe, Distinctive image features from scale-invariant key points, International journal of computer vision, 60(2), 91-110(2004).
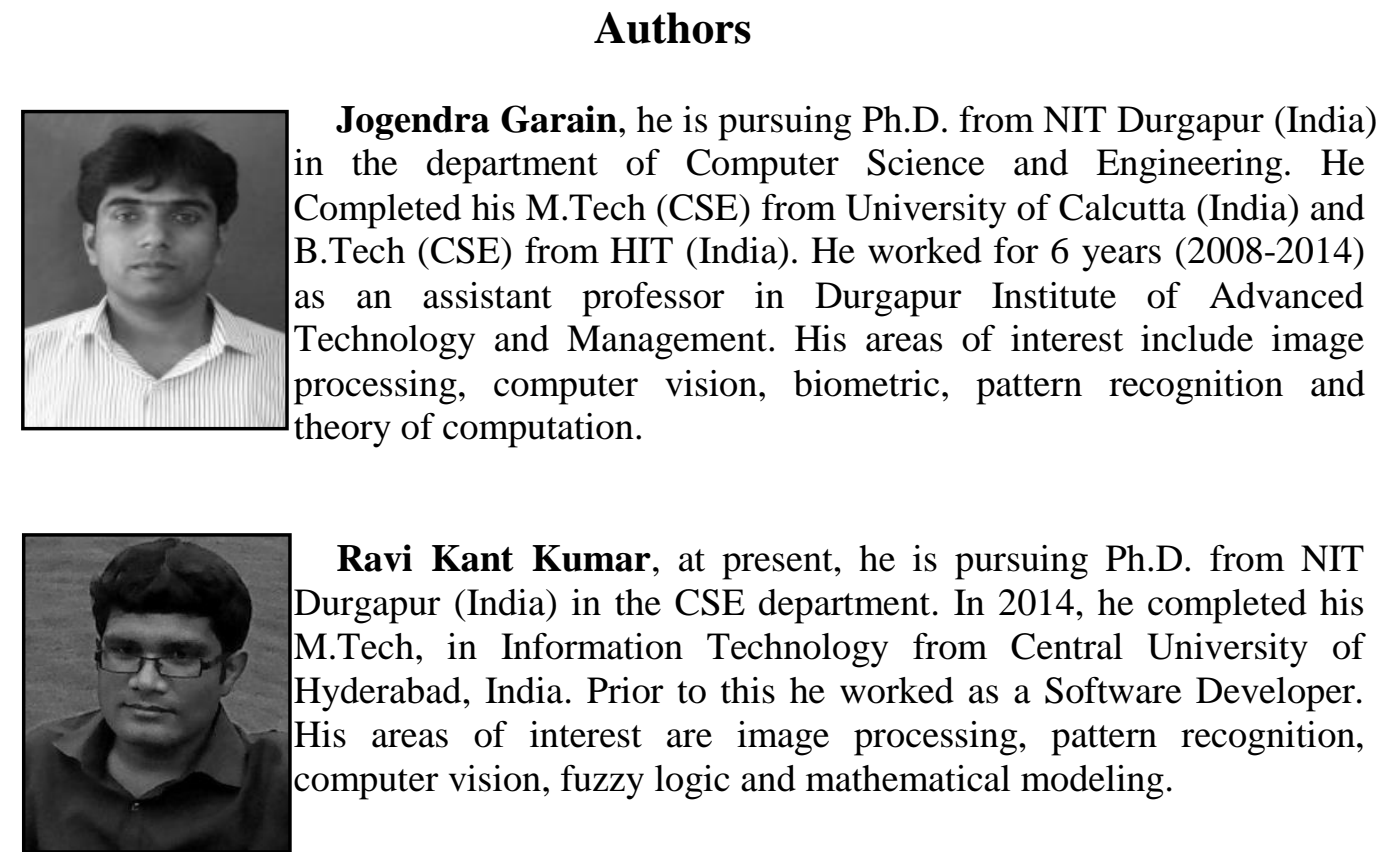

Ravi Kant Kumar, at present, he is pursuing Ph.D. from NIT Durgapur (India) in the CSE department. In 2014, he completed his M.Tech, in Information Technology from Central University of Hyderabad, India. Prior to this he worked as a Software Developer. His areas of interest are image processing, pattern recognition, computer vision, fuzzy logic and mathematical modeling.

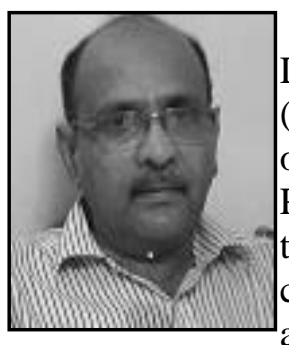

Prof. Goutam Sanyal, is designated as Professor in Department of Computer Science and Engineering and Dean (FW) of NIT Durgapur (India). His qualifications are Bachelors of Engineering (B.E), Masters of technology (M.Tech), Ph.D.(Engineering), FIE(India), MIEEE. Dr. Sanyal has more than 150 research papers in the reputed international journals and conferences. He has a work experience of 28 years in teaching and research and along with $\mathrm{PhD}$ Guidance. His areas of interest are Computer Architecture, Computer Graphics, Computer Vision, Image Processing, VLSI, and Mathematical Modeling. 


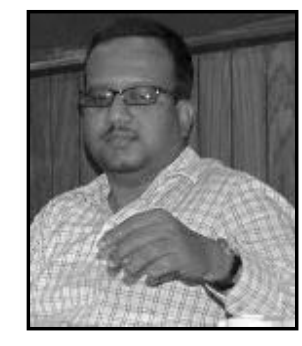

Dr. Dakshina Ranjan Kisku, is designated as an assistant Professor in the Department of CSE, NIT Durgapur (India). His qualifications are B.E, M.E and Ph.D. (Engineering) from Jadavpur University. He has about 12 years of teaching experience. Dr. Kisku has more than 38 research papers in the reputed international journals and conferences. His areas of research are Biometric, Computer Vision, Pattern Classification, Affective Computing and Data Compression. 